Europäische Zentralbank

\section{Wie steht es um den digitalen Euro?}

Digitale Zahlungen sind auf dem Vormarsch. Bargeld als einziges der breiten Öffentlichkeit zugängliches Zentralbankgeld verliert seit Jahren an Bedeutung. Zwar wurden 2019 in der Eurozone immer noch fast $50 \%$ des Transaktionsvolumens mit Bargeld transferiert, allerdings ist diese Tendenz, auch getrieben durch das im Zuge der Corona-Pandemie angepasste Bezahlverhalten, stark rückläufig (EZB, 2020b). Gleichzeitig könnte der potenzielle Markteintritt von privaten Stablecoins, die an eine nationale Währung, einen Währungskorb oder andere Vermögenswerte gebunden sind, zu einem weiteren Bedeutungsverlust von Zentralbankgeld im Zahlungsverkehr führen. Da die meisten Stablecoins zu 100\% durch Fiat-Währungen gedeckt sind, handelt es sich dabei nicht um neue Währungen, sondern um eine neue technische Infrastruktur, über die Fiat-Währungen, wie der Euro oder der US-Dollar, transferiert werden können. Laut einer aktuellen Studie der Bank für Internationalen Zahlungsausgleich (BIZ) denken deshalb $86 \%$ der befragten Zentralbanken darüber nach, eigene digitale Zentralbankwährungen (Central Bank Digital Currency: CBDC) einzuführen (Boar und Wehrli, 2021). Während sich bereits $14 \%$ der CBDC-Projekte, darunter in China und in Schweden, im Pilot-Stadium befinden, sind die CBDC-Bemühungen der Europäischen Zentralbank (EZB) noch verhaltener.

Im Oktober 2020 äußerte sich die EZB erstmals konkret darüber, wie eine europäische $\mathrm{CBDC}$, ein digitaler Euro, aussehen könnte. In ihrem „Report zum digitalen Euro“ beschreibt die EZB Szenarien, die sich sowohl auf die Erfüllung der Kernfunktionen der EZB als auch auf übergeordneten Ziele der EU beziehen (EZB, 2020a). Mit der Veröffentlichung des Reports befragte die EZB auch Bürger:innen und Unternehmen zu dem Vorhaben. Nach ersten Ergebnissen nannten $41 \%$ der Befragten Datenschutz als wichtigste Eigenschaften eines digitalen Euros (EZB, 2021). Anonymität sollte also beim Design des digitalen Euros eine große Rolle spielen. Nur wenn eine CBDC wie Bargeld auch anonyme Zahlungen ermöglicht, ist sie in der Lage, ein bargeldähnliches Equivalent in einer digitalen Welt bereitzustellen und die Bedeutung von Zentralbankgeld zu stärken.

Ob die EZB tatsächlich ein Projekt zum digitalen Euro starten wird, will sie erst Mitte 2021 entscheiden. Trotzdem hat EZBPräsidentin Lagarde bereits mit konkreten Aussagen zum Vorhaben überrascht. Sie betonte, dass der digitale Euro kommen werde und sie die Einführung in den nächsten fünf Jahren

(c) Der/die Autor:in(nen) 2021. Open Access: Dieser Artikel wird unter der Creative Commons Namensnennung 4.0 International Lizenz veröffentlicht (creativecommons.org/licenses/by/4.0/deed.de).

Open Access wird durch die ZBW - Leibniz-Informationszentrum Wirtschaft gefördert. erwarte (Siedenbiedel, 2021). China begann dagegen bereits 2014 mit ersten CBDC-Analysen. Bis zum Start der fortgeschrittenen Systemtests 2020 hat es sechs Jahre gedauert. Auch Schweden beschäftigt sich schon seit 2017 mit einer „e-Krona“. Der langwierige Prozess deutet darauf hin, dass es sich auch beim digitalen Euro um ein komplexes Unterfangen handelt - zu unklar sind derzeit noch die Implikationen auf den Finanzsektor und makroökonomische Auswirkungen. Aus diesem Grund ist eine gewisse Vorsicht der EZB durchaus verständlich. Allerdings muss auch betont werden, dass Schweden und China einen deutlichen Vorsprung haben. Wir schätzen den Vorsprung Chinas auf vier bis sechs Jahre.

Für die EZB stehen zunächst interne Analysen an, um grundsätzliche Anforderungen an das Design des digitalen Euros abzustecken. Daraus lassen sich erste Schlussfolgerungen für den Einfluss einer CBDC auf den Finanzsektor ableiten. Um eine Disruption des Finanzsektors zu verhindern, steht z. B. ein CBDC-Haltelimit (Der Spiegel, 2021) oder eine zweistufige Verzinsung (Bindseil, 2020) im Raum. Je attraktiver der digitale Euro für Endnutzer:innen letztlich sein wird, desto höher wird der negative Effekt auf Bankeinlagen ausfallen. Auch die technische Umsetzung des digitalen Euros ist noch unklar. Möglich wäre die Nutzung einer DistributedLedger-Technologie (DLT), die auch programmierbare Zahlungen ermöglichen würde, etwa in Form von Mikrozahlungen oder von Maschine-zu-Maschine-Zahlungen, die durch die zunehmende Digitalisierung von Geschäftsvorfällen immer mehr von der Industrie nachgefragt werden. Allerdings scheint die EZB momentan die Nutzung einer DLT nicht zu priorisieren, deshalb ist hier der private Finanzsektor gefragt.

\section{Literatur}

Bindseil, U. (2020), Tiered CBDC and the financial system, ECB Working Paper Series, 2351.

Boar C. und A. Wehrli (2021), Ready, steady, go? - Results of the third BIS survey on central bank digital currency, BIS Working Papers, 114.

Der Spiegel (2021), EZB: Warum die Zentralbank den digitalen Euro vorantreibt, https://www.spiegel.de/wirtschaft/ezb-warum-die-zentralbank-den-digitalen-euro-vorantreibt-a-a96283d5-c77f-4cd48191-2c0980d83b5b (2. März 2021).

EZB (2020a), Report on a digital euro, https://www.ecb.europa.eu/pub/ pdf/other/Report_on_a_digital_euro 4d7268b458.en.pdf\#page $=4$ (2. März 2021).

EZB (2020b), Study on the payment attitudes of consumers in the euro area (SPACE), https://www.ecb.europa.eu/pub/pdf/other/ecb. spacereport202012 bb2038bbb6.en.pdf (2. März 2021).

EZB (2021), ECB digital euro consultation ends with record level of public feedback, https://www.ecb.europa.eu/press/pr/date/2021/html/ecb. pr210113 ec9929f446.en.html (2. März 2021).

Siedenbiedel, C. (2021), EZB-Präsidentin kündigt an: Digitaler Euro spätestens in fünf Jahren, FAZ, 13. Januar, https://www.faz.net/aktuell/ finanzen/ezb-praesidentin-lagarde-kuendigt-digitalen-euro-in-fuenfjahren-an-17144352.html (2. März 2021).

Philipp Sandner, Jonas Groß, Lena Grale Frankfurt School of Finance \& Management jonas.gross@fs-blockchain.de 